\title{
Smoothness and singularities of the perfect form and the second Voronoi compactification of $\mathcal{A}_{g}$
}

\author{
Mathieu Dutour Sikirić, Klaus Hulek and Achill Schürmann
}

\begin{abstract}
We study the cones in the first Voronoi or perfect cone decomposition of quadratic forms with respect to the question which of these cones are basic or simplicial. We then deduce that the singular locus of the moduli stack $\mathcal{A}_{g}^{\text {Perf }}$, the toroidal compactification of the moduli space of principally polarized abelian varieties of dimension $g$ given by this decomposition, has codimension 10 if $g \geqslant 4$. Moreover, we describe the non-simplicial locus in codimension 10 . We also show that the second Voronoi compactification $\mathcal{A}_{g}^{\text {Vor }}$ has singularities in codimension 3 for $g \geqslant 5$.
\end{abstract}

\section{Introduction}

Reduction theory of quadratic forms is a classical topic. This leads to interesting admissible $\mathrm{GL}_{g}(\mathbb{Z})$-invariant tessellations of the rational closure of the space of positive-definite real quadratic forms in dimension $g$, also known as admissible rational polyhedral decompositions or fans. In algebraic geometry these give rise to toroidal compactifications of the moduli space $\mathcal{A}_{g}$ of principally polarized abelian varieties. This theory was first developed by Ash, Mumford, Rapoport and Tai [AMRT75] and has since been used by numerous authors in geometric studies of moduli of abelian varieties, where it is often essential not only to consider the non-compact spaces $\mathcal{A}_{g}$, but also to work with good projective models. More precisely, such a decomposition defines both a variety and a stack (see [FC90, SB06]). Toroidal compactifications have the property that the boundary is "big", that is, has codimension 1. All toroidal compactifications map surjectively onto the Satake compactification $\mathcal{A}_{g}^{\text {Sat}}$, which is set-theoretically given by

$$
\mathcal{A}_{g}^{\mathrm{Sat}}=\mathcal{A}_{g} \sqcup \mathcal{A}_{g-1} \sqcup \cdots \sqcup \mathcal{A}_{0} .
$$

In the literature three different types of decompositions have been studied in detail: the first Voronoi or perfect cone decomposition, the second Voronoi decomposition and the central cone decomposition, leading to the corresponding toroidal compactifications $\mathcal{A}_{g}^{\text {Perf }}, \mathcal{A}_{g}^{\text {Vor }}$ and $\mathcal{A}_{g}^{\text {Igu }}$, respectively; see [Nam80] for more details. In recent years the meaning of these various toroidal compactifications has been clarified. The central cone compactification has been identified with

Received 5 September 2013, accepted in final form 21 March 2015.

2010 Mathematics Subject Classification 14K10 (primary), 14D23, 14M25, 11H55 (secondary).

Keywords: abelian varieties, Siegel modular varieties, toroidal compactifications, Voronoi reduction theories.

This journal is (C) Foundation Compositio Mathematica 2015. This article is distributed with Open Access under the terms of the Creative Commons Attribution Non-Commercial License, which permits non-commercial reuse, distribution, and reproduction in any medium, provided that the original work is properly cited. For commercial re-use, please contact the Foundation Compositio Mathematica.

The first author has been supported by the Croatian Ministry of Science, Education and Sport under contract 098-0982705-2707 and by the Humboldt Foundation. The second author gratefully acknowledges support by DFG grant $\mathrm{Hu} 337 / 6-2$ and the Fund for Mathematics to the Institute of Advanced Study in Princeton, which provided excellent working conditions. 


\section{PERFECT FORM COMPACTIFICATIONS}

the Igusa compactification, a blow-up of the Satake compactification $\mathcal{A}_{g}^{\text {Sat }}$. The second Voronoi compactification $\mathcal{A}_{g}^{\text {Vor }}$ has a meaningful interpretation in terms of moduli of abelian varieties. Its boundary points correspond to degenerate abelian varieties, more precisely to semi-abelic varieties. For details the reader is referred to work by Alexeev [Ale02] and Olsson [Ols08]. Finally, the first Voronoi or perfect cone compactification is a good model to work with from the point of view of the classification theory of higher-dimensional algebraic varieties. Shepherd-Barron [SB06] has proved that $\mathcal{A}_{g}^{\text {Perf }}$ has canonical singularities and that its canonical bundle is ample if $g \geqslant 12$. Thus $\mathcal{A}_{g}^{\text {Perf }}$ is a canonical model in the sense of the minimal model program if $g \geqslant 12$.

The construction of toroidal compactifications of the moduli space $\mathcal{A}_{g}$ very roughly works as follows. One first has to choose an admissible rational polyhedral decomposition of the rational closure of the space of positive definite symmetric $g \times g$ matrices. This defines a compactification $\mathcal{A}_{g}^{\text {tor }}$ of $\mathcal{A}_{g}$ as a compact analytic space by adding a stratum to each cone in the chosen decomposition (where strata of $\mathrm{GL}_{g}(\mathbb{Z})$-equivalent cones will be identified). The codimension of the stratum which is added equals the dimension of the cone. In the cases we have mentioned above the compactification $\mathcal{A}_{g}^{\text {tor }}$ is in fact a projective variety. Naturally, the geometric properties of $\mathcal{A}_{g}^{\text {tor }}$ depend essentially on the properties of the chosen decomposition.

In this note we are especially interested in the singularities of toroidal compactifications. The singularities of the variety given by a fan arise in two different ways. First of all, the symplectic group $\operatorname{Sp}(2 g, \mathbb{Z})$ has torsion (different from \pm id). This gives rise to finite quotient singularities in $\mathcal{A}_{g}$ (unless the torsion element is a reflection, which happens only in genus $g=2$ ). Similarly, such quotient singularities can arise in the boundary of a toroidal compactification due to nonneatness of $\operatorname{Sp}(2 g, \mathbb{Z})$. Such quotient singularities are well behaved from an algebraic-geometric point of view and can for many considerations be neglected: if one replaces the group $\operatorname{Sp}(2 g, \mathbb{Z})$ by a principal congruence subgroup $\Gamma(\ell)=\{g \in \operatorname{Sp}(2 g, \mathbb{Z}) \mid g \equiv \mathbf{1} \bmod \ell\}$, then for $\ell \geqslant 3$ this group is neat and in particular torsion free. Hence the corresponding level cover $\mathcal{A}_{g}(\ell)$, respectively $\mathcal{A}_{g}^{\text {tor }}(\ell)$, will not acquire such singularities. A second type of singularities arises from "bad" behavior of cones $\sigma$ in the chosen decomposition. Recall that a cone $\sigma$ whose general element has rank $g$ is called basic if the reduced integral generators of its 1-dimensional faces can be completed to a $\mathbb{Z}$-basis of $\operatorname{Sym}^{2}\left(\mathbb{Z}^{g}\right)$. By an integrally reduced generator we mean a generator whose entries have gcd equal to 1 . Whenever we speak about integral generators we will from now on assume them to be integrally reduced. A cone is called simplicial if its rational generators can be completed to a $\mathbb{Q}$-basis, in other words, if the generators are linearly independent. If a cone $\sigma$ is basic, then its corresponding stratum lies in the non-singular locus of $\mathcal{A}_{g}^{\text {tor }}(\ell)$ for $\ell \geqslant 3$, whereas simplicial cones give rise to finite quotient singularities by abelian groups, the group being the quotient of the lattice $\operatorname{Sym}^{2}\left(\mathbb{Z}^{g}\right)$ by the lattice spanned by the integral generators of the cone. Non-simplicial cones give rise to more general singularities. Taking a level cover will not remove singularities which arise from non-basic cones; these are then singularities of the corresponding stack. We thus obtain open substacks $\mathcal{A}_{g \text {,smooth }}^{\text {tor }} \subset \mathcal{A}_{g \text {,simp }}^{\text {tor }} \subset \mathcal{A}_{g}^{\text {tor }}$ given by the partial compactification defined by the basic and simplicial singularities, respectively.

Knowledge of the singularities of a toroidal compactification $\mathcal{A}_{g}^{\text {tor }}$ and its level covers is obviously of geometric interest. One reason is that singularities are crucial for the understanding of the birational geometry of these varieties; another is that they are also important in order to understand topological properties. One example for the latter is the work by Grushevsky, Tommasi and the second author [GHT] on stable cohomology of $\mathcal{A}_{g}^{\text {Perf }}$. The relevance of $\mathcal{A}_{g \text {,smooth }}^{\text {Perf }}$ (and also $\mathcal{A}_{g \text {,simp }}^{\text {Perf }}$ ) in this context is that Poincaré duality holds here. For $g \leqslant 3$ the first and second Voronoi decomposition as well as the central cone decomposition all coincide and all cones 


\section{Dutour Sikirić, K. Hulek and A. Schürmann}

are basic. Hence the stacks are smooth in genus $\leqslant 3$. This changes in genus 4 . All cones in the second Voronoi decomposition are still basic, but this is no longer the case in the first Voronoi or perfect cone decomposition, which in genus 4 coincides with the central cone decomposition. Here, there is one non-basic cone, namely the perfect cone of the root lattice $\mathrm{D}_{4}$, which has dimension 10 and 12 rays, hence is neither basic nor simplicial. This defines the unique singular point in the 10 -dimensional stack $\mathcal{A}_{4}^{\text {Perf }}$. This also means that for $g \geqslant 4$ the stack $\mathcal{A}_{g}^{\text {Perf }}$ will always be singular in codimension 10 (or less). There is no a priori reason that the codimension of the singular locus of $\mathcal{A}_{g}^{\text {Perf }}$ could not be less than 10 for $g>4$. Our aim is to show that this is not the case. We shall also classify the non-simplicial locus in codimension 10; as it turns out, it all comes from the root lattice $\mathrm{D}_{4}$.

From the point of view of tessellations of the rational closure of the cone of positive-definite real quadratic forms, our results show the following: with respect to the properties basic and simplicial, the perfect cone decomposition is much more uniform up to dimension 9 than one might hope for in view of the explosion of arithmetically inequivalent perfect forms from dimension 6 onwards. The only exception to this in dimension 10 is given by the cone of the root lattice $\mathrm{D}_{4}$, which emphasizes once more the extraordinary importance of this root lattice.

THEOREM 1.1. (i) Every cone of dimension at most 9 in the perfect cone decomposition is basic, so the integral generators can be completed to a $\mathbb{Z}$-basis of $\operatorname{Sym}^{2}\left(\mathbb{Z}^{g}\right)$.

(ii) With the exception of the cone of the root lattice $\mathrm{D}_{4}$, every cone in the perfect cone decomposition of dimension at most 10 is simplicial, so its integral generators can be completed to a $\mathbb{Q}$-basis of $\operatorname{Sym}^{2}\left(\mathbb{Q}^{g}\right)$.

Form this we immediately obtain the following result.

Corollary 1.2. The stack $\mathcal{A}_{g}^{\text {Perf }}$ is smooth for $g \leqslant 3$ and the codimension of both the singular and the non-simplicial substack of $\mathcal{A}_{g}^{\text {Perf }}$ is 10 if $g \geqslant 4$.

Before we give the proof of Theorem 1.1 we note the following consequence for the intersection cohomology of the variety $\mathcal{A}_{g}^{\text {Perf }}$.

Corollary 1.3 ([GHT]). In degree $k \leqslant 10$ the intersection cohomology of the variety $\mathcal{A}_{g}^{\text {Perf }}$ is isomorphic to its singular cohomology:

$$
\mathrm{IH}^{k}\left(\mathcal{A}_{g}^{\text {Perf }}\right) \cong \mathrm{H}^{k}\left(\mathcal{A}_{g}^{\text {Perf }}\right) \quad \text { for } k \leqslant 10 .
$$

We also answer the same question for the second Voronoi compactification.

Theorem 1.4. (i) For $g \leqslant 4$ every cone in the second Voronoi compactification is basic.

(ii) For $g \geqslant 5$ there are non-simplicial cones in dimension 3, thus for these dimensions $\mathcal{A}_{g}^{\text {Vor }}$ is singular in codimension 3 .

Our results lead to natural further questions which we will pose as problems at the end of Sections 3, 4 and 5 .

\section{Proof of Theorem 1.1}

Background on perfect cones. Let us start with some background on the perfect cone decomposition. For a vector $v \in \mathbb{Z}^{g}$ we write $p(v)=v v^{t}$ for the corresponding rank 1 matrix. Any cone $\sigma$ 


\section{PERFECT FORM COMPACTIFICATIONS}

of the perfect cone decomposition is of the form $\sum_{i=1}^{M} \mathbb{R}_{+} p\left(v_{i}\right)$, where $v_{1}, \ldots, v_{M} \in \mathbb{Z}^{g}$. More precisely, perfect cones arise in this way if and only if the $v_{i}$ are coordinate vectors (with respect to a lattice basis) of all the minimal vectors for some $g$-dimensional lattice. Here, minimal vectors come in pairs $\pm v_{i}$ and because $p\left(v_{i}\right)=p\left(-v_{i}\right)$, it suffices to consider one coordinate vector for each of them. Moreover, by the minimal vector property the gcd of the coordinates is 1 for each $v_{i}$. So the corresponding generators $p\left(v_{1}\right), \ldots, p\left(v_{M}\right)$ of the perfect cone $\sigma$ are integrally reduced (that is, their gcd is 1, too). For further details on perfect cones (which are sometimes called Voronoi domains) and the associated theory of perfect quadratic forms, we refer to [Mar03] and [Sch09].

By $\operatorname{dim} \sigma$ we denote the dimension of a cone $\sigma$ in $\operatorname{Sym}^{2}\left(\mathbb{Z}^{g}\right)$, that is, the dimension of its linear hull. The faces of perfect cones (intersections with a supporting hyperplane) are perfect cones of lower dimensions themselves. For a given $g$, the perfect cones give a face-to-face tessellation of the rational closure of the space of real, positive-definite $g \times g$ matrices. This tessellation is invariant with respect to the action $M \mapsto U M U^{t}$ of the group $\mathrm{GL}_{g}(\mathbb{Z})$ on this space. Two perfect cones are called $\mathrm{GL}_{g}(\mathbb{Z})$-equivalent if they are in the same orbit with respect to this group action. By a classical theorem due to Voronoi, we know that there exist only finitely many perfect cones up to equivalence for every fixed $g$. For a given $g$ it is therefore possible, at least in principle, to classify all perfect cones $\sigma$ of a given dimension $\operatorname{dim} \sigma=N$. However, for a classification of all perfect cones of dimension $N$, hence in spaces $\operatorname{Sym}^{2}\left(\mathbb{Z}^{g}\right)$ with varying $g$, we need an additional argument.

Reduction to finitely many perfect cones. Let us take a cone $\sigma$ of dimension $N$ of the perfect cone decomposition, generated by $p\left(v_{1}\right), \ldots, p\left(v_{M}\right)$. Denote by $d$ the dimension of the lattice $L=\mathbb{Z} v_{1}+\cdots+\mathbb{Z} v_{M}$ in $\mathbb{Z}^{g}$. Then $L$ is a finite index sublattice of the lattice $L^{\prime}=(L \otimes \mathbb{R}) \cap \mathbb{Z}^{g}$ having the same affine span. The lattice $L^{\prime}$ has a $\mathbb{Z}$-basis $\left\{w_{1}, \ldots, w_{d}\right\}$ that can be extended to a $\mathbb{Z}$-basis $\left\{w_{1}, \ldots, w_{g}\right\}$ of $\mathbb{Z}^{g}$. Thus, by applying a suitable $\mathrm{GL}_{g}(\mathbb{Z})$-mapping (base change for $\mathbb{Z}^{g}$ ), we may assume that $L^{\prime}$ is equal to $\mathbb{Z}^{d}$. As a consequence, the properties that concern us, namely that the family $\left(p\left(v_{i}\right)\right)_{1 \leqslant i \leqslant M}$ is extensible to a $\mathbb{Z}$ - or $\mathbb{Q}$-basis, can be considered by assuming $d=g$. That is, we may assume that $L$ is a full-dimensional, finite-index sublattice of $\mathbb{Z}^{g}$. Since $p\left(v_{1}\right), \ldots, p\left(v_{g}\right)$ are linearly independent if $v_{1}, \ldots, v_{g}$ are linearly independent, we may assume $g \leqslant \operatorname{dim} \sigma$. This leaves only a finite number of cases to consider when classifying all perfect cones of a given dimension $N$.

Proof of Theorem 1.1(i). Unfortunately, we are not aware of a general method for proving basicness of perfect cones without a full enumeration. So for the proof of (i) we have to get a hand on all 9-dimensional perfect cones for all $g \leqslant 9$ and check for each one of the cones that it is basic. Note that given the reduced integral generators $p\left(v_{1}\right), \ldots, p\left(v_{M}\right)$ of a fixed perfect cone, we can easily check basicness computationally, for instance by considering the generators themselves as vectors and checking whether their Gram matrix has determinant 1.

We split the needed classification of 9-dimensional perfect cones into three parts:

- For $g \leqslant 7$ all perfect cones have been classified up to $\mathrm{GL}_{g}(\mathbb{Z})$-equivalence in [EVGS13].

- For $g=8$ we computationally classify all 9-dimensional cones in Lemma 4.1 below.

- For $g=9$ the perfect cones of dimension 9 have to be simplicial, as we show in Lemma 3.1 in the next section. Therefore, these perfect cones are in 1-to-1 correspondence with sets of linearly independent vector pairs $\pm v_{1}, \ldots, \pm v_{9} \in \mathbb{Z}^{9}$ that are coordinates (with respect to a lattice basis) of all minimal vectors for some 9-dimensional lattice. Such vector configurations were classified in [KMS12], giving us 31 perfect cones of dimension 9 for $g=9$, 


\section{Dutour Sikirić, K. Hulek and A. Schürmann}

corresponding to the entries with $s=r=s^{\prime}=9$ of Tables 2, 3 and 7 in [KMS12].

In addition to the summarized information given here, we provide a complete list of all 9dimensional perfect cones in electronic form on the webpage [DS]. Representatives of the cones $\sigma$ of the perfect decomposition of dimension at most 10 and genus $g \leqslant 9$ are given there. The classification in genus $g=8,9$ and dimension 10 was obtained by extensions of the method of Section 4. Table 1 gives the number of orbits of cones.

TABLE 1. Number of orbits of cones in the perfect cone decomposition for $g \leqslant 9$ and dimension at most 10 .

\begin{tabular}{|c||c|c|c|c|c|c|c|}
\hline $\mathrm{g} \downarrow, \operatorname{dim} \rightarrow$ & 4 & 5 & 6 & 7 & 8 & 9 & 10 \\
\hline \hline 4 & 1 & 3 & 4 & 4 & 2 & 2 & 2 \\
\hline 5 & & 2 & 5 & 10 & 16 & 23 & 25 \\
\hline 6 & & & 3 & 10 & 28 & 71 & 162 \\
\hline 7 & & & & 6 & 28 & 115 & 467 \\
\hline 8 & & & & & 13 & 106 & 783 \\
\hline 9 & & & & & & 44 & 759 \\
\hline
\end{tabular}

Proof of Theorem 1.1(ii). For our proof of assertion (ii) we "only" have to consider the 10dimensional perfect cones, because from (i) we know that all cones of dimension at most 9 are simplicial (as they are basic, which is stronger). It seems quite challenging to classify all 10dimensional perfect cones, so our proof relies on a combination of mathematical reasoning and computer assisted checks:

- For $g \leqslant 7$ we can use the previously known classification of perfect cones again. According to [EVGS13] there are 656 inequivalent 10-dimensional perfect cones and we checked computationally that all of them are simplicial, except the one of the root lattice $\mathrm{D}_{4}$.

On the webpage $[\mathrm{DS}]$ we provide a complete list of all 10-dimensional perfect cones with $g \leqslant 7$.

- For $g=8, g=9$ and $g=10$ there is no complete classification of 10-dimensional perfect cones known so far. Here, we use our Lemma 3.1 below. It shows for general $g$, that all perfect cones of dimension $g, g+1$ and $g+2$ are necessarily simplicial.

Classifying 10-dimensional perfect cones? A complete classification of 10-dimensional perfect cones appears to be highly challenging, if not out of reach at the moment. Although an extension of our method to prove Lemma 4.1 could possibly be feasible (to deal with the case $g=9$ ), the necessary extension of the work in [KMS12] to the case $g=10$ seems computationally hardly realizable. A slightly simpler task could be the case $g=8$. More generally, enumerating the number of inequivalent $(8+k)$-dimensional perfect cones for small $k$ could be feasible for $g=8$. Lemma 4.1 shows that there are 106 classes for $k=1$. Since this number is much less than the 10916 classes of full-dimensional perfect cones for $g=8$, which have been classified in [DSSV07], one may hope to obtain a complete list for $k=2$ as well.

Note that only with the help of our Lemma 3.1 below could we avoid this very difficult (currently impossible) classification of 10-dimensional perfect cones, in order to prove part (ii) of the theorem. On the other hand, for part (i) of the theorem, our proof relies on the complete classification of all 9-dimensional perfect cones, which would have been extremely painful (if not 


\section{PERFECT FORM COMPACTIFICATIONS}

impossible) without computer assistance. So, it is just this special combination of mathematical reasoning and computer assistance which allowed us to obtain the results of this paper.

\section{Low-dimensional perfect cones are simplicial}

Perfect cones for $g=2$ and $g=3$ are known to be simplicial. In fact, in these two cases there exists only one class of top-dimensional perfect cones of dimension 3 and 6 , respectively. Both are associated with the root lattice $\mathrm{A}_{g}$ and are known to be simplicial. Since all perfect cones are faces of top-dimensional perfect cones and since faces of simplicial cones are simplicial, we see that perfect cones of any dimension $N$ are simplicial for $g=2$ and $g=3$. This argument does not work for $g \geqslant 4$ as, for instance, the top-dimensional perfect cones associated with the root lattices $\mathrm{D}_{g}$ are not simplicial in these cases. The following lemma shows, however, that perfect cones are simplicial for any $g$ in case their dimension is sufficiently small, namely at most $g+2$.

LEMMA 3.1. Let $\sigma=\mathbb{R}_{+} p\left(v_{1}\right)+\cdots+\mathbb{R}_{+} p\left(v_{M}\right)$ be a cone of the perfect cone decomposition of $\operatorname{Sym}^{2}\left(\mathbb{Z}^{g}\right)$. Assume that $\left(v_{1}, \ldots, v_{M}\right)$ span $\mathbb{R}^{g}$ and that $\operatorname{dim} \sigma=g, g+1$ or $g+2$. Then $\sigma$ is simplicial.

Proof. As mentioned above, the cases $g=2$ and $g=3$ are known by classical results. So we may assume $g \geqslant 4$ and that the lemma is true for all dimensions less than $g$.

Among the generators of $\sigma$ we can find $g$ linearly independent vectors $\left(v_{i}\right)_{1 \leqslant i \leqslant g}$ that determine a basis of $\mathbb{Q}^{g}$. By using this basis, the vector space spanned by $\left(p\left(v_{i}\right)\right)_{1 \leqslant i \leqslant g}$ can be identified with the space of diagonal $g \times g$ matrices. That is, after a suitable base change, we may assume that the $v_{i}$ are the standard basis vectors. Given an arbitrary vector $v=\sum_{i=1}^{g} \alpha_{i} v_{i}$, we see that $p(v)$ is linearly independent of the generators $p\left(v_{i}\right)$ if and only if $\alpha_{i} \neq 0$ for at least two indices $i_{1} \neq i_{2}$, since the non-diagonal entry $\left(i_{1}, i_{2}\right)$ is non-zero in that case.

Suppose $\operatorname{dim} \sigma=g$. Then any additional generator $p(v)$ of a perfect cone would belong to the vector space spanned by the $\left(p\left(v_{i}\right)\right)_{1 \leqslant i \leqslant d}$. Therefore, by the argument above, $v=\sum_{i=1}^{g} \alpha_{i} v_{i}$ with $\alpha_{i} \neq 0$ for just one $\alpha_{i}$. Hence, $v$ is a multiple of one $v_{i}$ in that case, and therefore $p(v)$ is a multiple of $p\left(v_{i}\right)$ as well, showing that $p(v)$ is not an additional generator.

Suppose $\operatorname{dim} \sigma=g+1$. Then we can find a vector $v$ in $L=\mathbb{Z} v_{1}+\cdots+\mathbb{Z} v_{g}$ with $p(v)$ a generator of $\sigma$ linearly independent of the $p\left(v_{i}\right)$. After suitable scaling of the vectors $v_{i}$ we may assume without loss of generality that $v=\sum_{i=1}^{r} v_{i}$ with $2 \leqslant r \leqslant g$. Suppose now that $w=\sum_{i=1}^{g} \alpha_{i} v_{i}$ with $p(w) \in \sigma$. Then there exist $\gamma$ and $\beta_{i}$ such that $p(w)=\gamma p(v)+\sum_{i=1}^{g} \beta_{i} p\left(v_{i}\right)$ and we obtain the equation

$$
\left(\sum_{i=1}^{g} \alpha_{i} x_{i}\right)^{2}=\gamma\left(\sum_{i=1}^{r} x_{i}\right)^{2}+\sum_{j=1}^{g} \beta_{i} x_{i}^{2}
$$

for the corresponding quadratic forms. From this it follows that we cannot have $\alpha_{i} \alpha_{j} \neq 0$ for some $i \geqslant r+1$ and $i \neq j$. This implies that either (i) $\alpha_{i_{0}} \neq 0$ for only one $i_{0} \geqslant r+1$ or (ii) $\alpha_{i}=0$ for all $i \geqslant r+1$.

(i) In the first case we deduce $w=\alpha_{i_{0}} v_{i_{0}}$ from (3.1). Hence $p(w)$ is a multiple of $p\left(v_{i_{0}}\right)$ in that case and therefore $p(w)$ cannot be an additional generator of $\sigma$.

(ii) In the second case we deduce from (3.1) that $\alpha_{i} \alpha_{j}=\gamma$ for all $i, j \leqslant r$ with $i \neq j$. In particular, $\alpha_{i} \neq 0$ for all $i \leqslant r$.

In the case $r=2$ we have $\beta_{i}=0$ for $i \geqslant 3$ because of $\alpha_{i}=0$ for all $i \geqslant 3$. Thus the putative generator $p(w)$ is actually in the 3 -dimensional vector space spanned by the generators 


\section{Dutour Sikirić, K. Hulek And A. Schürmann}

$p(v), p\left(v_{1}\right), p\left(v_{2}\right)$. We know from the already solved case of dimension $g=2$ that $p(w)$ must be a multiple of one of the other three generators if they span a perfect cone themselves. Every face of a perfect cone is a perfect cone, so it remains to verify that $p(v), p\left(v_{1}\right), p\left(v_{2}\right)$ span a face of the perfect cone $\sigma$. That is, we have to check whether or not there exists a linear form on $\operatorname{Sym}^{2}\left(\mathbb{Z}^{g}\right)$ that evaluates to 0 on the three generators and is positive on the remaining ones. Taking the inner product with $p\left(v_{3}\right)+\cdots+p\left(v_{g}\right)$ gives such a linear form.

So suppose $r \geqslant 3$. Then at least two of the $\alpha_{i}$ with $i \leqslant r$ are of the same sign and so $\gamma>0$. So, all $\alpha_{i}$ with $i \leqslant r$ are of the same sign, which we may assume to be positive. For all subsets $S=\left\{i_{1}, i_{2}, i_{3}\right\}$ of $\{1, \ldots, r\}$ with distinct indices $i_{1}, i_{2}, i_{3}$ the equations $\alpha_{i_{1}} \alpha_{i_{2}}=\alpha_{i_{1}} \alpha_{i_{3}}=$ $\alpha_{i_{2}} \alpha_{i_{3}}=\gamma$ have the unique solution $\alpha_{i_{1}}=\alpha_{i_{2}}=\alpha_{i_{3}}=\sqrt{\gamma}$. So $w$ is actually a multiple of $v$, showing again that $p(w)$ cannot be an additional generator of $\sigma$.

Suppose $\operatorname{dim} \sigma=g+2$. By a suitable scaling of the $v_{i}$ we can find vectors of the form $v=\sum_{i=1}^{r} v_{i}$ and $v^{\prime}=\sum_{i=1}^{g} \lambda_{i} v_{i}$ such that the set $\left\{p\left(v_{1}\right), \ldots, p\left(v_{g}\right), p(v), p\left(v^{\prime}\right)\right\}$ consists of generators of $\sigma$ which form a basis of the rational vector space spanned by $\sigma$. Assume that $p(w)$ is an element of this vector space and let $w=\sum_{i=1}^{g} \mu_{i} v_{i}$. Then there exist $\alpha_{i}, \beta, \gamma$ such that in terms of quadratic forms we have

$$
\sum_{i=1}^{g} \alpha_{i} x_{i}^{2}+\beta\left(\sum_{i=1}^{r} x_{i}\right)^{2}+\gamma\left(\sum_{i=1}^{g} \lambda_{i} x_{i}\right)^{2}=\left(\sum_{i=1}^{g} \mu_{i} x_{i}\right)^{2} .
$$

By the arguments for the case $\operatorname{dim} \sigma=g+1$ we may assume $\beta \neq 0$ and $\gamma \neq 0$. We distinguish two cases: (i) $r<g$ and (ii) $r=g$.

(i) Assume $r<g$. Then (3.2) implies $\gamma \lambda_{i} \lambda_{g}=\mu_{i} \mu_{g}$ for all $i<g$.

If $\lambda_{g}=0$, then $\mu_{i} \mu_{g}=0$. If $\mu_{g} \neq 0$, then $\mu_{i}=0$ for all $i<g$ and $w$ is a multiple of $v_{g}$. If $\mu_{g}=0$, then we have necessarily $\alpha_{g}=0$ and the problem is reduced to a lower-dimensional one.

If $\lambda_{g} \neq 0$, then (3.2) implies $\mu_{g} \neq 0$. So $\lambda_{i}=\mu_{i} \kappa$ with $\kappa=\mu_{g} /\left(\gamma \lambda_{g}\right)$ for $i<g$. Hence, the quadratic forms $p\left(v^{\prime}\right)$ and $p(w)$ are multiples of each other, showing that $p(w)$ cannot be an additional generator of $\sigma$.

(ii) Assume $r=g$. Then from (3.2) we deduce the equality $\beta+\gamma \lambda_{i} \lambda_{j}=\mu_{i} \mu_{j}$ for all $i \neq j$. We may assume that all $\lambda_{i}$ and $\mu_{i}$ are non-zero, since otherwise we can permutate between $v, v^{\prime}$ and $w$ and reduce to the preceding case (i). By a suitable scaling of the $v_{i}$ we may assume $\mu_{g}=1$. So we get $\mu_{i}=\beta+\gamma \lambda_{i} \lambda_{g}$ and for $1 \leqslant i<j<g$,

$$
\begin{aligned}
0 & =\mu_{i} \mu_{j}-\beta-\gamma \lambda_{i} \lambda_{j}=\left(\beta+\gamma \lambda_{i} \lambda_{g}\right)\left(\beta+\gamma \lambda_{j} \lambda_{g}\right)-\beta-\gamma \lambda_{i} \lambda_{j} \\
& =\beta^{2}-\beta+\beta \gamma \lambda_{g}\left(\lambda_{i}+\lambda_{j}\right)+\gamma\left(\gamma \lambda_{g}^{2}-1\right) \lambda_{i} \lambda_{j}=a+b\left(\lambda_{i}+\lambda_{j}\right)+c \lambda_{i} \lambda_{j} .
\end{aligned}
$$

If $a c \neq b^{2}$, then there exists a fractional function $\phi: \mathbb{P}^{1}(\mathbb{R}) \rightarrow \mathbb{P}^{1}(\mathbb{R})$ such that $\lambda_{i}=\phi\left(\lambda_{j}\right)$ and $\phi \circ \phi=\mathrm{Id}$. It follows that there are one or two possible values for $\lambda_{i}$, and in case of two different values, we find for any pair $(i, j)$ with $1 \leqslant i<j<g$ that $\lambda_{i}$ is equal to one value and $\lambda_{j}$ is equal to the other. The restriction $g \geqslant 4$ implies that $\lambda_{i}$ takes only one value in this case.

If $a c=b^{2}$, then we have $c \neq 0$ and $\left(\lambda_{i}+b / c\right)\left(\lambda_{j}+b / c\right)=0$. So, no two $\lambda_{i}$ with $i<g$ can be different from $-b / c$. If there is one index $i_{0}<g$ with $\lambda_{i_{0}} \neq-b / c$ and if $\lambda_{g} \neq-b / c$ as well, we may choose a different special index than $g$ and obtain a contradiction too. Otherwise, if $\lambda_{g}=-b / c$, we may choose $i_{0}$ as this special index. So we may assume $\lambda_{i}=-b / c$ for all $i<g$. If all the $\lambda_{i}$ are equal (hence also $\lambda_{g}=-b / c$ ), then $v$ and $v^{\prime}$ are multiples of each other, contradicting the assumption that $p(v)$ and $p\left(v^{\prime}\right)$ are linearly independent. 


\section{PeRfeCt FORM COMPACTIFICATIONS}

So, we have $\lambda_{g} \neq-b / c, \lambda_{i}=\lambda_{1}$ and $\mu_{i}=\mu_{1}$ for $i<g$. As we assume $g \geqslant 4$, setting $\sum_{i=1}^{g-1} x_{i}=0$ in (3.2) yields $\alpha_{i}=0$ for $i<g$. Using the two variables $y_{1}=\sum_{i=1}^{g-1} x_{i}$ and $y_{2}=x_{g}$, we see that (3.2) is actually a relation between forms of two variables. Therefore, the putative generator $p(w)$ of $\sigma$ is in the 3-dimensional vector space spanned by the generators $p\left(v_{g}\right), p(v)$, $p\left(v^{\prime}\right)$. We know from the already solved case of dimension $g=2$ that $p(w)$ must be a multiple of one of the other three generators if these span a perfect cone themselves. As in the proof for the case $\operatorname{dim} \sigma=g+1$ (subcase (ii) with $r=2$ ), this follows if we show that $p\left(v_{g}\right), p(v), p\left(v^{\prime}\right)$ span a face of the perfect cone $\sigma$. To see this we may construct a linear form on $\operatorname{Sym}^{2}\left(\mathbb{Z}^{g}\right)$ that evaluates to 0 on the three generators and is positive on the remaining ones. Such a form is for instance obtained by considering the inner product with the projection of $p\left(v_{1}\right)+\cdots+p\left(v_{g-1}\right)$ onto the linear space orthogonal to the span of the three generators $p\left(v_{g}\right), p(v)$ and $p\left(v^{\prime}\right)$.

Based on the enumeration results that we have obtained, it seems plausible that simpliciality also occurs in case $\operatorname{dim} \sigma=g+3$ or $g+4$.

\section{Classification of 9-dimensional perfect cones}

As mentioned in Section 2, for a complete classification of 9-dimensional perfect cones, we need to obtain the full list of all such cones for the case $g=8$.

LEMma 4.1. There are 106 orbits of 9-dimensional cones in the perfect cone decomposition for $g=8$.

Proof. Our classification is based on a computer-assisted case distinction. The full list can be obtained from the webpage [DS]. Here, we briefly describe the necessary ingredients and our computational steps.

Using our simplicity lemma. By Lemma 3.1 all 9-dimensional perfect cones for $g=8$ are necessarily simplicial, that is, of the form $\mathbb{R}_{+} p\left(v_{1}\right)+\cdots+\mathbb{R}_{+} p\left(v_{9}\right)$, with linearly independent generators $p\left(v_{1}\right), \ldots, p\left(v_{9}\right)$. As we assume $g=8$, without loss of generality we may assume that $\left(v_{1}, \ldots, v_{8}\right)$ are linearly independent too.

Knowing a priori that the 9-dimensional perfect cones are simplicial is a quite strong condition. It in particular implies that all its facets (codimension 1 faces) are simplicial 8-dimensional perfect cones. Thus we may assume that $\pm v_{1}, \ldots, \pm v_{8} \in \mathbb{Z}^{8}$ are the coordinates (with respect to a lattice basis) of all minimal vectors for some 8-dimensional lattice. Such sets of eight minimal vector pairs have been classified by Martinet in [Mar01] (see entries satisfying $n=s=r=s^{\prime}=8$ in Tableau 11.1). Up to $\mathrm{GL}_{8}(\mathbb{Z})$-equivalence there are 13 such sets of vectors and we may assume that $\left(v_{1}, \ldots, v_{8}\right)$ is a representative for one of these 13 orbits.

Reduction to a finite enumeration. If $L$ denotes the sublattice spanned by the vectors $\left(v_{1}\right.$, $\left.\ldots, v_{8}\right)$ in $\mathbb{Z}^{8}$, then from Martinet's classification [Mar01] we know that the index $i(L)$ in $\mathbb{Z}^{8}$ satisfies $1 \leqslant i(L) \leqslant 5$. We may assume that $\left(v_{1}, \ldots, v_{8}\right)$ is of maximal index among all possible 8-subsets of $\left(v_{1}, \ldots, v_{9}\right)$.

Given a fixed set of vectors $\left(v_{1}, \ldots, v_{8}\right)$, we derive some restrictive conditions for the additional vectors $v_{9} \in \mathbb{Z}^{8}$ from this maximality assumption. For $j=1, \ldots, 8$ we define the lattice

$$
L_{j}=\mathbb{Z} v_{1}+\cdots+\mathbb{Z} v_{j-1}+\mathbb{Z} v_{j+1}+\cdots+\mathbb{Z} v_{8}+\mathbb{Z} v_{9} .
$$




\section{Dutour Sikirić, K. Hulek and A. Schürmann}

If $L_{j}$ is full-dimensional, then its index $i\left(L_{j}\right)$ in $\mathbb{Z}^{8}$ is at most $i(L)$ by our maximality assumption for $L$. Thus

$$
\left|\operatorname{det}\left(v_{1}, \ldots, v_{j-1}, v_{j+1}, \ldots, v_{8}, v_{9}\right)\right| \in\{0,1, \ldots, i(L)\},
$$

with the determinant being 0 if and only if $L_{j}$ is not full-dimensional. By using the exterior product, we can rewrite these determinant conditions as $-i(L) \leqslant\left\langle w_{j}, v_{9}\right\rangle \leqslant i(L)$ with $w_{j}$ a fixed integral vector, depending only on the vectors $v_{1}, \ldots, v_{j-1}, v_{j+1}, \ldots, v_{8}$ (orthogonal to it). Thus for a fixed set of vectors $\left(v_{1}, \ldots, v_{8}\right)$ we obtain eight pairs of linear inequalities for the possible additional integral vector $v_{9}$. Geometrically, these conditions define a parallelepiped and we can use for instance the program zsolve from [4ti2] to enumerate its integral points.

Note that the eight determinant conditions (4.1) have altogether $(1+2 i(L))^{8}$ possible values. It is easy to see that the matrix $\left(w_{j}\right)_{j=1, \ldots, 8}$ is the adjugate matrix of $\left(v_{i}\right)_{i=1, \ldots, 8}$, and so is of determinant $i(L)^{7}$. This implies that the number of integral solutions $v_{9}$ is actually reasonable, allowing zsolve to find all solutions. So the condition that the lattice spanned by $\left\{v_{1}, \ldots, v_{8}\right\}$ be of maximal index is a key assumption for the enumeration to work.

Exploiting symmetry. We are left with a finite number of possible sets $\left\{v_{1}, \ldots, v_{9}\right\}$. Using Algorithm 1 of [KMS12], we can test for each such set whether it defines a 9-dimensional perfect cone or not. However, each of these tests is computationally very expensive and it is therefore advisable to consider further reductions beforehand, in order to finish the classification.

A large reduction of cases can be obtained from using the symmetry within a given configuration $\mathcal{V}=\left( \pm v_{1}, \ldots, \pm v_{8}\right)$. We only need to consider vectors $v_{9}$ up to the automorphism group (a subgroup of $\mathrm{GL}_{8}(\mathbb{Z})$ ) of the configuration $\mathcal{V}$. Once we have this integral automorphism group of $\mathcal{V}$, we identify orbits of possible extensions $v_{9}$ and choose only one representative for each orbit to proceed.

To compute the automorphism group, we first consider the group $G_{2}$ of rational automorphisms of $\mathcal{V}$, which is the hyperoctahedral group of size $2^{8} 8$ !. In order to obtain the integral automorphisms of $\mathcal{V}$, we first determine a subgroup $G_{1}$ formed by transpositions $(i, j)$ and sign changes $\left(v_{i} \mapsto-v_{i}\right)$ which induce integral automorphisms. The full automorphism group within $\mathrm{GL}_{8}(\mathbb{Z})$ is then obtained by iterating over double cosets $G_{1} h G_{1}$ and keeping the ones that preserve $\mathcal{V}$.

Treatment of the remaining tuples. For each set $\left\{v_{1}, \ldots, v_{9}\right\}$ that remains we check whether each of its 8-subsets is integrally equivalent to one of the 13 orbits classified by Martinet in [Mar01]. This is a necessary condition, as every facet of the potential perfect cone has to be a perfect cone itself. If all these checks are positive, we test whether the candidate $\left\{v_{1}, \ldots, v_{9}\right\}$ defines a 9-dimensional perfect cone by using Algorithm 1 of [KMS12].

We thus get 131 systems of 9 vectors to test for unimodular equivalence. This gives 106 systems, respectively orbits, under $\mathrm{GL}_{8}(\mathbb{Z})$-equivalence.

The computation was dominated by the realizability tests using Algorithm 1 of [KMS12].

Open problem. There are examples of cones which are simplicial but not basic. One such example is given by the shortest vectors of the dual root lattice $E_{7}^{*}$ (the index of the corresponding sublattice of $\operatorname{Sym}^{2}\left(\mathbb{Z}^{7}\right)$ is 384). Our classification of 9-dimensional perfect cones shows that perfect cones are simplicial and basic in low dimensions. Note, however, that this is not the case for general polyhedral cones. If we take $v_{1}=(1,1)$ and $v_{2}=(1,-1)$ for instance, then the relation

$$
\frac{1}{2}\left(p\left(v_{1}\right)+p\left(v_{2}\right)\right)=p((1,0))+p((0,1))
$$




\section{PERFECT FORM COMPACTIFICATIONS}

shows that the cone spanned by $\left\{p\left(v_{1}\right), p\left(v_{2}\right)\right\}$ is simplicial but not basic, since the two generators cannot be extended to a $\mathbb{Z}$-basis of $\operatorname{Sym}^{2}\left(\mathbb{Z}^{2}\right)$. Of course, $\left\{ \pm v_{1}, \pm v_{2}\right\}$ cannot be realized as the set of shortest vector of a 2-dimensional lattice, so $\left\{p\left(v_{1}\right), p\left(v_{2}\right)\right\}$ does not generate a perfect cone. In contrast, for perfect cones and $g \leqslant 9$, our classification shows that if $\left\{v_{1}, \ldots, v_{g}\right\}$ are independent vectors of $\mathbb{Z}^{g}$ such that $\left\{p\left(v_{1}\right), \ldots, p\left(v_{g}\right)\right\}$ are the generators of a perfect cone, then the cone is simplicial and basic. Is this true for general $g$ ?

\section{Proof of Theorem 1.4}

Here we consider the singularities of the second Voronoi compactification $\mathcal{A}_{g}^{\text {Vor }}$. The second Voronoi decomposition is described in detail in [Sch09]. It is known only up to dimension 5 (see [Eng00]). For $g \leqslant 5$, a complete system of $\mathrm{GL}_{g}(\mathbb{Z})$-inequivalent polyhedral cones in $\operatorname{Sym}^{2}\left(\mathbb{Z}^{g}\right)$ can be accessed electronically using the program [SV], for instance.

For the proof of Theorem 1.4(i) one can simply check whether or not all polyhedral cones for $g \leqslant 4$ are basic. For this it is even enough to check whether the top-dimensional cones of the second Voronoi decomposition are basic. There is only one such cone each for $g=2$ and $g=3$ and there are three cones for $g=4$, as already observed by Voronoi (see [Vor08, Vor09]). We do not know whether Voronoi checked them for basicness, but he could certainly have done so. Recall that basicness of a cone follows if the Gram matrix of its generators has determinant 1.

All cones of dimension 1 or 2 are spanned by one or two extremal rays and are thus trivially simplicial. Thus for the proof of statement (ii) of Theorem 1.4, it suffices to find a non-simplicial cone of dimension 3 for $g \geqslant 5$. Using [Eng00] we find two cones of dimension 3 that are spanned by 4 generators and so are non-simplicial. They are:

$$
\begin{gathered}
\left(\begin{array}{rrrrr}
4 & -2 & -2 & 0 & -2 \\
-2 & 4 & 0 & -1 & 1 \\
-2 & 0 & 4 & -1 & 1 \\
0 & -1 & -1 & 3 & -1 \\
-2 & 1 & 1 & -1 & 3
\end{array}\right), \quad\left(\begin{array}{rrrrrr}
2 & -1 & -1 & 0 & -1 \\
-1 & 2 & 0 & 0 & 0 \\
-1 & 0 & 2 & -1 & 1 \\
0 & 0 & -1 & 2 & -1 \\
-1 & 0 & 1 & -1 & 2
\end{array}\right) \\
\left(\begin{array}{rrrrrr}
2 & -1 & -1 & 0 & -1 \\
-1 & 2 & 0 & -1 & 1 \\
-1 & 0 & 2 & 0 & 0 \\
0 & -1 & 0 & 2 & -1 \\
-1 & 1 & 0 & -1 & 2
\end{array}\right), \quad\left(\begin{array}{lllll}
0 & 0 & 0 & 0 & 0 \\
0 & 0 & 0 & 0 & 0 \\
0 & 0 & 0 & 0 & 0 \\
0 & 0 & 0 & 1 & -1 \\
0 & 0 & 0 & -1 & 1
\end{array}\right)
\end{gathered}
$$

and

$$
\begin{aligned}
& \left(\begin{array}{rrrrr}
3 & -1 & -1 & -1 & -1 \\
-1 & 4 & -1 & -1 & 0 \\
-1 & -1 & 3 & 1 & -1 \\
-1 & -1 & 1 & 3 & -1 \\
-1 & 0 & -1 & -1 & 4
\end{array}\right), \quad\left(\begin{array}{rrrrrr}
6 & -2 & -2 & -2 & -2 \\
-2 & 6 & -1 & -1 & 0 \\
-2 & -1 & 4 & 1 & -1 \\
-2 & -1 & 1 & 4 & -1 \\
-2 & 0 & -1 & -1 & 6
\end{array}\right) \\
& \left(\begin{array}{rrrrr}
2 & 0 & -1 & -1 & -1 \\
0 & 2 & -1 & -1 & 0 \\
-1 & -1 & 2 & 1 & 0 \\
-1 & -1 & 1 & 2 & 0 \\
-1 & 0 & 0 & 0 & 2
\end{array}\right), \quad\left(\begin{array}{rrrrr}
5 & -1 & -2 & -2 & -2 \\
-1 & 4 & -1 & -1 & 0 \\
-2 & -1 & 3 & 1 & 0 \\
-2 & -1 & 1 & 3 & 0 \\
-2 & 0 & 0 & 0 & 4
\end{array}\right)
\end{aligned}
$$




\section{Dutour Sikirić, K. Hulek and A. Schürmann}

The two polyhedral cones spanned by these generators can be extended to spaces $\operatorname{Sym}^{2}\left(\mathbb{Z}^{g}\right)$ for any $g>5$ and so the result follows.

Open problem. We note that we did not find any polyhedral cone of index greater than 1 in the second Voronoi decomposition. It appears to be an open problem whether or not for every $g$, all cones in the second Voronoi decomposition have generators of its 1-dimensional faces that generate an integral lattice in $\operatorname{Sym}^{2}\left(\mathbb{Z}^{g}\right)$ of index 1 .

\section{ACKNOWLEDGEMENTS}

We thank the referee for useful remarks which helped to improve the presentation of the text.

\section{REFERENCES}

Ale02 V. Alexeev, Complete moduli in the presence of semiabelian group action, Ann. of Math. (2) 155 (2002), no. 3, 611-708; http://dx.doi.org/10.2307/3062130.

AMRT75 A. Ash, D. Mumford, M. Rapoport and Y. Tai, Smooth compactification of locally symmetric varieties, Lie Groups: History, Frontiers and Applications, vol. 6, Math. Sci. Press, Brookline, Mass., 1975.

DS M. Dutour Sikirić, A list of all 9-dimensional perfect forms, available at http://mathieudutour. altervista.org/PerfectCones/.

DSSV07 M. Dutour Sikirić, A. Schürmann and F. Vallentin, Classification of eight-dimensional perfect forms, Electron. Res. Announc. Amer. Math. Soc. 13 (2007), 21-32; http://dx.doi.org/10.1090/ S1079-6762-07-00171-0.

Eng00 P. Engel, The contraction types of parallelohedra in $E^{5}$, Acta Cryst. Sect. A 56 (2000), no. 5, 491-496; http://dx.doi.org/10.1107/S0108767300007145.

EVGS13 P. Elbaz-Vincent, H. Gangl and C. Soulé, Perfect forms, K-theory and the cohomology of modular groups, Adv. Math. 245 (2013), 587-624; http://dx.doi.org/10.1016/j.aim.2013.06.014.

FC90 G. Faltings and C.-L. Chai, Degeneration of abelian varieties (with an appendix by David Mumford), Ergeb. Math. Grenzgeb. (3), vol. 22, Springer-Verlag, Berlin, 1990; http://dx.doi. org/10.1007/978-3-662-02632-8.

GHT S. Grushevsky, K. Hulek and O. Tommasi, Stable cohomology of the perfect cone toroidal compactification of the moduli space of abelian varieties, J. reinie angew. Math., to appear, arXiv:1307.4646.

KMS12 W. Keller, J. Martinet and A. Schürmann, On classifying Minkowskian sublattices (with an appendix by Mathieu Dutour Sikirić), Math. Comp. 81 (2012), no. 278, 1063-1092; http://dx. doi.org/10.1090/S0025-5718-2011-02528-7.

Mar01 J. Martinet, Sur l'indice d'un sous-réseau (with an appendix by Christian Batut), Réseaux Euclidiens, Designs Sphériques et Formes Modulaires, 163-211, Monogr. Enseign. Math., vol. 37, Enseignement Math., Geneva, 2001.

Mar03 _ Perfect lattices in Euclidean spaces, Grundlehren Math. Wiss., vol. 327, SpringerVerlag, Berlin, 2003; http://dx.doi.org/10.1007/978-3-662-05167-2.

Nam80 Y. Namikawa, Toroidal compactification of Siegel spaces, Lecture Notes in Math., vol. 812, Springer, Berlin, 1980; http://dx.doi.org/10.1007/BFb0091051.

Ols08 M. C. Olsson, Compactifying moduli spaces for abelian varieties, Lecture Notes in Math., vol. 1958, Springer-Verlag, Berlin, 2008; http://dx.doi.org/10.1007/978-3-540-70519-2.

SB06 N. I. Shepherd-Barron, Perfect forms and the moduli space of abelian varieties, Invent. Math. 163 (2006), no. 1, 25-45; http://dx.doi.org/10.1007/s00222-005-0453-0. 


\section{PERFECT FORM COMPACTIFICATIONS}

Sch09 A. Schürmann, Computational geometry of positive definite quadratic forms: polyhedral reduction theories, algorithms, and applications, Univ. Lecture Ser., vol. 48, Amer. Math. Soc., Providence, RI, 2009; http://dx.doi.org/10.1090/ulect/048.

SV A. Schürmann and F. Vallentin, scc (secondary cone cruiser) - a C++-implementation of Voronoi's reduction theory based on the classification of Delone triangulations, available at http://www.math.uni-magdeburg.de/lattice_geometry/.

4ti2 The 4ti2 team, A software package for algebraic, geometric and combinatorial problems on linear spaces, available at http://www.4ti2.de.

Vor08 G. Voronoi, Nouvelles applications des paramètres continus à la théorie des formes quadratiques. Deuxième mémoire. Recherches sur les parallélloèdres primitifs, J. Reine Angew. Math. 134 (1908), 198-287; http://dx.doi.org/10.1515/crll.1908.134.198.

Vor09_, Nouvelles applications des paramètres continus à la théorie des formes quadratiques. Deuxième mémoire. Recherches sur les parallélloèdres primitifs, J. Reine Angew. Math. 136 (1909), 67-181; http://dx.doi.org/10.1515/crll.1909.136.67.

Mathieu Dutour Sikirić mathieu.dutour@gmail.com

Rudjer Bosković Institute, Bijenicka 54, 10000 Zagreb, Croatia

Klaus Hulek hulek@math.uni-hannover.de Institut für Algebraische Geometrie, Welfengarten 1, 30167 Hannover, Germany

Achill Schürmann achill.schuermann@uni-rostock.de Universität Rostock, Institute of Mathematics, 18051 Rostock, Germany 